\title{
Twenty-year experience of lung transplantation at a single center: Influence of recipient diagnosis on long-term survival
}

\author{
Marc de Perrot, MD \\ Cecilia Chaparro, MD \\ Karen McRae, MD \\ Thomas K. Waddell, MD \\ Denis Hadjiliadis, MD \\ Lianne G. Singer, MD \\ Andrew F. Pierre, MD \\ Michael Hutcheon, MD \\ Shaf Keshavjee, MD
}

From the Toronto Lung Transplant Program, Toronto General Hospital, University of Toronto, Toronto, Ontario, Canada.

Read at the Eighty-third Annual Meeting of The American Association for Thoracic Surgery, Boston, Mass, May 4-7, 2003.

Received for publication May 2, 2003; revisions requested Oct 29, 2003; accepted for publication Nov 1, 2003.

Address for reprints: S. Keshavjee, MD, Director, Toronto Lung Transplant Program, Toronto General Hospital, 200 Elizabeth St, EN 10-224, Toronto, M5G 2C4 Ontario, Canada (E-mail: shaf.keshavjee@ uhn.on.ca).

J Thorac Cardiovasc Surg 2004;127: 1493-501

$0022-5223 / \$ 30.00$

Copyright $\odot 2004$ by The American Association for Thoracic Surgery

doi:10.1016/j.jtcvs.2003.11.047
Objectives: The objective of this study was to examine the long-term patient outcomes of lung transplantation in a single center.

Methods: Between 1983 and 2003, 521 lung transplants were performed in 501 patients. Major indications were cystic fibrosis $(\mathrm{n}=124)$, chronic obstructive pulmonary disease $(\mathrm{n}=88)$, alpha-1 antitrypsin deficiency $(\mathrm{n}=63)$, pulmonary fibrosis $(n=97)$, primary pulmonary hypertension $(n=35)$, Eisenmenger syndrome $(n=21)$, and miscellaneous end-stage lung diseases $(n=93)$.

Results: The 5-, 10-, and 15-year survivals for all recipients were $55.1 \%$ (95\% confidence interval: $\pm 5 \%), 35.3 \%( \pm 6 \%)$, and $26.5 \%( \pm 11 \%)$, respectively. The most common causes of death were sepsis and bronchiolitis obliterans syndrome. Despite an increased postoperative mortality rate, patients with primary pulmonary hypertension achieved the best long-term survival (10-year survival: 59\%). Recipients with cystic fibrosis without Burkholderia cepacia infection achieved significantly better long-term survival (10-year survival: 52\%) than those with Burkholderia cepacia infection (10-year survival: $15 \%$ ). The 10-year survival was also significantly better in recipients with chronic obstructive pulmonary disease $(43 \%)$ than in recipients with alpha- 1 antitrypsin deficiency (23\%). Although the incidence of bronchiolitis obliterans syndrome was similar between recipients with chronic obstructive pulmonary disease (39\%) and alpha-1 antitrypsin deficiency (46\%), recipients with alpha-1 antitrypsin deficiency died of sepsis more frequently than recipients with chronic obstructive pulmonary disease (27\% vs $6 \%$, respectively; $P$ $=.0003)$.

Conclusions: Although bronchiolitis obliterans syndrome and sepsis still limit the durability of the benefit, lung transplantation returns many patients with end-stage lung disease to active and productive lives. Differences in the complications and long-term survival show the important contribution of the recipient diagnosis to the success of lung transplantation.

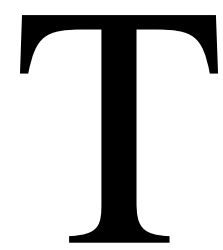

he first lung transplantation ever reported in a human was performed by Hardy at the University of Mississippi in 1963 for a patient with advanced stage bronchogenic carcinoma. ${ }^{1}$ The patient died of renal failure after 18 days. During the following 20 years, approximately 40 clinical lung allotransplants were performed, but only 1 patient was discharged from the hospital 8 months after the transplant procedure. This patient died a short time later of sepsis. ${ }^{2}$ In 1983, Patterson and 
colleagues from the Toronto General Hospital at the University of Toronto performed the first successful isolated single-lung transplant procedure, and in 1986 the same group described the first successful series of en bloc doublelung transplants. ${ }^{3,4}$ The technique of en bloc double-lung transplantation was supplanted by bilateral sequential lung transplantation at the end of 1989 , a procedure that became the standard and that has undergone minor changes since then. $^{5}$

Whereas the first decade of clinical lung transplantation focused on technical details of the transplant procedure, the second decade experienced improvements in techniques of lung preservation and postoperative management. Improvements in early morbidity and mortality have permitted a progressive extension of the list of indications for lung transplantation and gradually led to liberalization of the lung donor-selection criteria with a subsequent increase in the number of transplants performed each year.

This report reviews the 20-year experience of the Toronto Lung Transplant Program with particular emphasis on long-term outcomes.

\section{Material and Methods}

All lung transplants performed at the Toronto General Hospital between November 1983 and March 2003 were reviewed. In addition, physicians in our program have performed all pediatric lung transplants at the Hospital for Sick Children in Toronto since 1996; these patients were also included in the analysis.

\section{Recipient Selection}

Recipients were usually selected according to the guidelines outlined by the International Society for Heart and Lung Transplantation and the American Thoracic Society. ${ }^{6}$ However, the process of selection has gradually evolved over time. The first single-lung transplant was performed in a patient with pulmonary fibrosis in 1983, the first heart-lung transplant was performed in a patient with primary pulmonary hypertension in 1985 , and the first double-lung transplant was performed in a patient with alpha-1 antitrypsin deficiency in 1986 . Thereafter, the indications for singlelung transplant were extended to patients with sarcoidosis and eosinophilic granuloma in 1987, and double-lung transplants have been performed in patients with cystic fibrosis and bronchiectasis since 1988. More recently, lung transplantation has been performed for select malignant processes such as bronchioloalveolar carcinoma $(n=3)$ or metastatic leiomyoma $(n=1)$.

\section{Organ Allocation}

In Canada, all lung donors located in Ontario are allocated to Toronto. Donors from other provinces are allocated to the local program if there is one. If there is no program, the lungs are sequentially offered to the different lung transplant centers in the country. Transplant physicians from the selected transplant center then choose the recipient on the basis of blood group, size match, patient status, and time on the waiting list.

\section{Donor Management}

Our current donor management includes the administration of an intravenous bolus of steroids (methylprednisolone $15 \mathrm{mg} / \mathrm{kg}$ ) to all potential donors immediately after the declaration of brain death. An infusion of vasopressin (1-4 U/h) is generally used in donors receiving inotropes to taper the requirement for epinephrine and norepinephrine. Volume recruitment maneuvers (sighs) are performed regularly to prevent atelectasis and recruit alveoli. This maneuver is of particular importance after the apnea test, which predisposes the lungs to atelectasis. An arterial blood gas analysis with an $\mathrm{FIO}_{2}$ of 1.0 and a positive end-expiratory pressure of $5 \mathrm{~cm}$ $\mathrm{H}_{2} \mathrm{O}$ is repeated every 2 to 3 hours, and a chest radiograph is repeated every 3 to 4 hours. At the time of retrieval, the lungs are re-expanded by manual inflation to exclude areas of atelectasis. A final arterial blood gas analysis with an $\mathrm{FIO}_{2}$ of 1.0 and a positive end-expiratory pressure of $5 \mathrm{~cm} \mathrm{H}_{2} \mathrm{O}$ is then performed in the operating room. The final decision regarding use of the lungs is made based on a review of the donor history, the last arterial blood gas tension performed after the lungs have been fully re-expanded in the operating room, the chest radiograph appearance, bronchoscopic findings, and direct examination and palpation of the lungs at the time of retrieval.

\section{Lung Preservation}

The method of lung preservation has evolved from cold atelectatic immersion in the early experience to an anterograde flush with Euro-Collins solution in 1989. The preservation solution was then switched from Euro-Collins to low-potassium dextran $(50 \mathrm{~mL} / \mathrm{kg}$, Perfadex, Vitrolife, Goteborg, Sweden) in April 1998, and an in situ retrograde flush with 1 liter of Perfadex was added to the anterograde pulmonary artery flush in July 2000. A bolus of prostaglandin $\mathrm{E}_{1}(500 \mu \mathrm{g}$, Prostin VR, Upjohn, Don Mills, Ontario, Canada) is administered directly into the pulmonary artery immediately before initiating the pulmonary artery flush, and 500 $\mu \mathrm{g}$ of prostaglandin $\mathrm{E}_{1}$ is also added to the Perfadex solution. The current method of lung preservation used by our group has recently been described in detail elsewhere. ${ }^{7}$

\section{Transplant Procedure}

Single and bilateral sequential lung transplantation are performed in a standard fashion. Single-lung transplants are generally performed through a posterolateral thoracotomy. However, an anterolateral thoracotomy with transverse division of the sternum has been performed occasionally in patients with emphysema requiring lung-volume reduction on the contralateral side. Bilateral-lung transplants are performed through a bilateral anterolateral thoracotomy in the fourth intercostal space with oblique transverse division of the sternum. Initially, heart-lung transplants were performed through a midline sternotomy, but since 1995 a bilateral transverse thoracotomy has been used to take advantage of the better exposure to the posterior mediastinum and both pleural spaces. The lung with the least function, as judged by ventilationperfusion scan, is removed first. While the donor lung is prepared on the back table, care is taken to preserve the collateral blood supply to the donor bronchus from the pulmonary artery. Thus, limited dissection of peribronchial tissue and shortening of the bronchus up to 1 cartilage proximal to the upper lobe bronchial takeoff are routinely used. The bronchial anastomosis is performed 
with a running suture of 4-0 PDS (Ethicon, Markham, Canada) for the membranous part and interrupted sutures of 4-0 Prolene (Davis and Geck, Ontario, Canada) for the cartilaginous part. If the size discrepancy is favorable, we prefer to telescope the recipient bronchus into the donor bronchus. Theoretically, the recipient bronchus may then help to maintain an adequate bronchial lumen and act as an anastomotic stent if the donor bronchus becomes ischemic. In pediatric recipients, we usually perform end-to-end anastomosis with 4-0 PDS or 4-0 Vicryl (Ethicon, Markham, Canada) for the cartilaginous part because of the smaller size of the bronchus. In adults with small left main bronchus, an end-to-end anastomosis is occasionally performed with interrupted 4-0 Prolene. The pulmonary artery anastomosis is performed with 5-0 Prolene interrupted in 2 places, and the venous anastomosis is performed with a horizontal everting mattress running suture to decrease the risk of thrombogenic redundant tissue within the lumen of the left atrium. ${ }^{8}$

At the end of the procedure, the double-lumen tube is replaced with a single-lumen endotracheal tube, and the patient is bronchoscoped in the operating room to remove secretions and examine the bronchial anastomoses. Postoperatively, we routinely administer low-dose heparin (100 U/h) and Rheomacrodex (10\% Dex$\operatorname{tran} 40,500 \mathrm{~mL} / 24 \mathrm{~h}$ ) as an intravenous infusion for 7 days to improve the bronchial microcirculation.

Cardiopulmonary bypass (CPB) is used only when required. The usual indications for planned CPB are primary or secondary pulmonary hypertension disorders. CPB is otherwise instituted during the transplant procedure if the recipient does not tolerate unilateral clamping of the pulmonary artery or single-lung ventilation. The most common time for institution of $\mathrm{CPB}$ is after implantation of the first lung in a bilateral-lung transplant. Aprotinin has been used for all CPB procedures since 1994 to limit the risk of postoperative bleeding.

\section{Infection Prophylaxis}

Infection prophylaxis includes the administration of broad-spectrum antibiotics and antiviral therapy. Antiviral therapy is directed against cytomegalovirus (CMV). Since 1991 ganciclovir has been administered if the donor or the recipient has positive CMV serology. Hyperimmune globulin was also used in the highest risk group, that is, seronegative recipients receiving lungs from a seropositive donor. The length of time that intravenous ganciclovir is administered has been progressively reduced during the past 10 years in recipients with positive CMV serology and is currently limited to 2 weeks followed by oral ganciclovir for 12 weeks. Intravenous ganciclovir is still maintained for 3 months in the high-risk group, but the use of hyperimmune globulin has been discontinued for the past 4 years in that group. If both donors and recipients are seronegative, acyclovir is administered postoperatively as prophylaxis for Herpes simplex infections. Fungal prophylaxis has been used routinely only in the past 2 years during the in-hospital phase because of major construction occurring in our institution.

\section{Immunosuppression}

Immunosuppression has remained similar over the years and is based on cyclosporine, azathioprine, and corticosteroids. One dose of oral cyclosporine $(5 \mathrm{mg} / \mathrm{kg})$ is administered preoperatively immediately before surgery. Cyclosporine is then resumed postoperatively and administered orally or through the nasogastric tube ( $5 \mathrm{mg} / \mathrm{kg}$ twice daily). Cyclosporine levels of 250 to $350 \mathrm{ng} / \mathrm{mL}$ are targeted for the first 6 months and then tapered down. Patients receive azathioprine immediately postoperatively orally or through the nasogastric tube (1-2 $\mathrm{mg} / \mathrm{kg}$ daily). The steroids are started during the transplant procedure with 1 bolus (solumedrol $500 \mathrm{mg}$ ) given before reperfusion of the first allograft. Then intravenous solumedrol is started postoperatively $(0.5 \mathrm{mg} / \mathrm{kg}$ daily $)$ for 3 days followed by oral prednisone thereafter $(0.5 \mathrm{mg} / \mathrm{kg}$ daily $)$. Induction therapy was used for all recipients up to 1995 . Thereafter, it was discontinued, initially in recipients who tested positive for Burkholderia cepacia cystic fibrosis and then in everyone. Currently, patients with a high risk of renal failure (eg, after prolonged CPB) frequently receive $1 \mathrm{dose}(20 \mathrm{mg}$ ) of basiliximab (Simulect, Norvatis, Canada) on the first and fourth postoperative days to hold the cyclosporine for up to 5 to 10 days after the transplant procedure and to protect the kidney from cyclosporine toxicity.

\section{Acute Rejection}

The diagnosis of rejection episodes continues to be a challenge because the clinical signs are nonspecific and resemble those of infection, fluid overload, and ischemia-reperfusion injury. We perform routine transbronchial biopsies at 2 and 6 weeks, 3, 6, 9, 12,18 , and 24 months. In addition, transbronchial biopsy and bronchioloalveolar lavages are performed whenever clinically indicated.

\section{Airway Complications}

All airway complications such as dehiscence, stenosis, and malacia were recorded. Stenoses that did not require surgical interventions were considered "mild," whereas those requiring surgical interventions such as dilatation, stenting, or debridement were labeled "significant."

\section{Statistical Analysis}

Continuous variables are expressed as mean $\pm \mathrm{SD}$ or as median and range. Statistical comparisons of categoric variables were made by chi-square analysis or Fisher's exact test. Statistical comparisons of continuous variables were made by Student $t$ test or analysis of variance when necessary. Survival after surgery was analyzed by the Kaplan-Meier method. The evaluation of the differences between groups was conducted by the log-rank test. The variables analyzed were recipient age $(<18$ years, 18-39 years, 40-64 years, and $\geq 65$ years), recipient diagnosis (cystic fibrosis, emphysema, pulmonary fibrosis, pulmonary hypertension, and others), time of transplant (1983-1987, 1988-1992, 1993-1997, and 1998-2003), use of CPB (yes/no), and type of lung transplantation performed (single- vs bilateral-lung transplantation). Statistical analysis was performed using the GraphPad software package (GraphPad Software, Inc, San Diego, Calif).

\section{Results}

A total of 501 recipients underwent 521 transplantations, with 20 patients undergoing retransplantation. There were 396 bilateral sequential lung transplants, 13 en bloc doublelung transplants, 100 single-lung transplants, and 12 heart- 


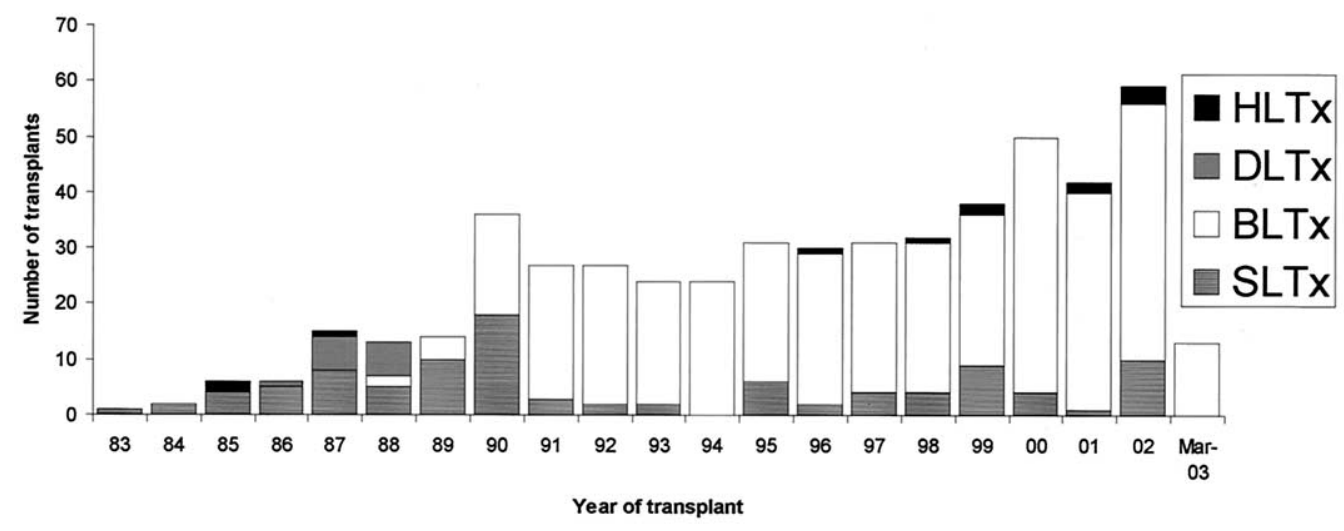

Figure 1. Number and type of transplant performed by year at the Toronto General Hospital. HLTx, Heart-lung transplant; DLTX, double-lung transplant; $B L T x$, bilateral-lung transplant; $S L T x$, single-lung transplant.

TABLE 1. Indications for lung transplantations by type of transplant

\begin{tabular}{lccc}
\hline & $\begin{array}{c}\text { Single lung } \\
\text { (N = 100) }\end{array}$ & $\begin{array}{c}\text { Bilateral lung } \\
(\mathbf{N}=\mathbf{3 9 6})\end{array}$ & $\begin{array}{c}\text { Heart-lung } \\
\text { (N = 12) }\end{array}$ \\
\hline Emphysema & $26(26 \%)$ & $125(32 \%)$ & $0(0 \%)$ \\
Pulmonary fibrosis & $45(45 \%)$ & $52(13 \%)$ & $0(0 \%)$ \\
Cystic fibrosis & $1(1 \%)$ & $122(31 \%)$ & $1(8 \%)$ \\
Pulmonary hypertension & $10(10 \%)$ & $37(9 \%)$ & $9(75 \%)$ \\
Retransplantation & $7(7 \%)$ & $12(3 \%)$ & $1(8 \%)$ \\
Other & $11(11 \%)$ & $48(12 \%)$ & $1(8 \%)$ \\
\hline
\end{tabular}

TABLE 2. Indications for transplantation by era

\begin{tabular}{lcccc}
\hline & $\begin{array}{c}1983-1990 \\
\text { (N = 93) }\end{array}$ & $\mathbf{1 9 9 1 - 1 9 9 4}$ & $\mathbf{1 9 9 5 - 1 9 9 8}$ & $\mathbf{1 9 9 9 - 2 0 0 3}$ \\
& $20(22 \%)$ & $41(40 \%)$ & $34(27 \%)$ & $56(28 \%)$ \\
\hline Emphysema & $23(25 \%)$ & $9(9 \%)$ & $19(16 \%)$ & $46(23 \%)$ \\
Pulmonary fibrosis & $18(19 \%)$ & $25(25 \%)$ & $34(27 \%)$ & $47(23 \%)$ \\
Cystic fibrosis & $(\mathbf{N}=\mathbf{2 0 2})$ \\
Pulmonary hypertension & $10(11 \%)$ & $10(10 \%)$ & $15(12 \%)$ & $21(10 \%)$ \\
Retransplantation & $8(9 \%)$ & $2(2 \%)$ & $4(3 \%)$ & $6(3 \%)$ \\
Other & $14(14 \%)$ & $15(14 \%)$ & $18(15 \%)$ & $26(13 \%)$ \\
\hline
\end{tabular}

lung transplants. The number of transplants has progressively increased since 1983, and since 1991, most have been bilateral sequential lung transplants (Figure 1).

A total of 263 patients were men, and 238 patients were women. The age of the recipients ranged from 8 to 71 years old with a median age of 46 years. A small group of recipients were at the extremes of age; 22 recipients were aged less than 18 years old, and 23 recipients were aged 65 years or more.

Of single-lung transplants, $45 \%$ were performed in patients with pulmonary fibrosis and $26 \%$ were performed in patients with emphysema. In contrast, bilateral-lung transplants were performed predominantly in patients with emphysema (32\%) and cystic fibrosis (31\%), and less fre- quently in patients with pulmonary fibrosis (13\%) (Table 1). Lung transplantation was performed in 56 patients with pulmonary hypertension. The majority of them underwent bilateral-lung transplantation $(\mathrm{n}=37), 10$ underwent single-lung transplantation, and 9 underwent heart-lung transplantation. Lung retransplantations were bilateral in 12 patients, limited to 1 side in 7 patients, and combined heart and lung in 1 patient.

The proportion of patients with emphysema, pulmonary fibrosis, cystic fibrosis, and pulmonary hypertension has not changed greatly over time despite a transient increase in the proportion of patients with emphysema in the early 1990s (Table 2). The proportion of lung retransplantations performed decreased from $9 \%$ before 1991 to $3 \%$ or less thereafter. We currently perform lung retransplantation primarily in patients with chronic graft dysfunction. In the early experience, lung retransplantation was also performed in patients with primary graft failure, but 3 of 4 patients undergoing retransplantation because of primary graft dysfunction died within 2 months of surgery, and therefore this indication was abandoned.

Overall, CPB was used in $35 \%$ of the patients who have undergone lung transplantation since 1991. CPB was used in essentially all patients with a diagnosis of primary pulmonary hypertension. However, CPB was used in $49 \%$ of patients with pulmonary fibrosis, in $26 \%$ of patients with cystic fibrosis, and in only $13 \%$ of patients with emphysema (Figure 2).

The proportion of patients experiencing airway complications decreased from $27 \%$ between 1983 and 1990 to $13 \%$ between 1991 and 1994, and to 9\% between 1999 and 2003 (Figure 3). During the last 4 years, only 20 of 371 bronchial anastomoses $(5.4 \%)$ presented with some degree of bronchial complications. Significant stenoses occurred in 11 cases and required serial dilatation $(n=6)$ and stent placement $(n=5)$. Debridement for excessive granulation tissue 


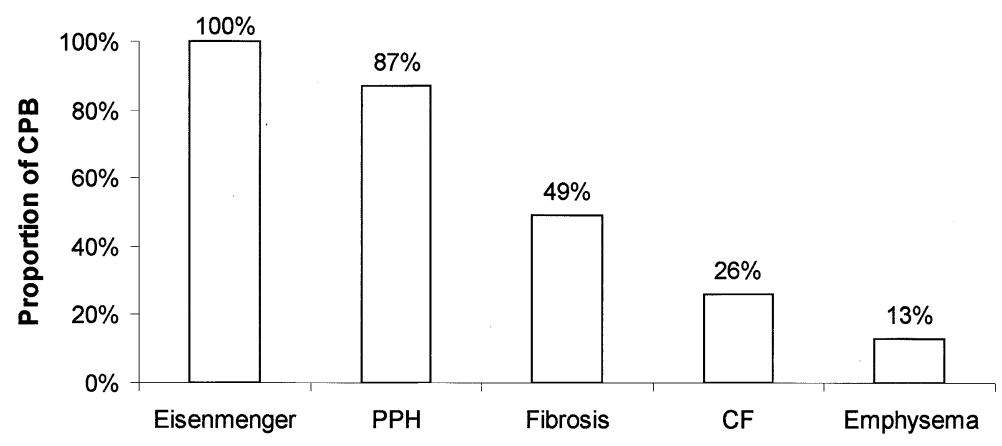

Figure 2. Proportion of transplantations performed with CPB since 1991. PPH, Primary pulmonary hypertension; $C F$, cystic fibrosis.

was required in 1 case. Mild stenosis was observed in 2 cases on flexible bronchoscopy, but both patients were clinically asymptomatic and did not require any treatment. Bronchomalacia was observed in 2 cases, and bronchial dehiscence occurred in 4 cases. Two dehiscences were partial and healed after conservative management. One dehiscence was located along the membranous part of the bronchus and was successfully treated with adequate percutaneous drainage, and 1 dehiscence occurred in a patient presenting with cardiac dysfunction after bilateral-lung transplantation. This patient eventually died in the postoperative course.

The overall 5-, 10-, and 15-year survivals for the entire cohort of patients were $55.1 \%$ (95\% confidence interval: $\pm 5 \%), 35.3 \%( \pm 6 \%)$, and $26.5 \%( \pm 11 \%)$, respectively (Figure 4). The best survival was achieved in recipients with primary pulmonary hypertension and in recipients with cystic fibrosis who tested negative for Burkholderia $(B)$ cepacia before the transplant procedure. The recipient group with $B$ cepacia negative cystic fibrosis had 5- and 10-year survivals of $76 \%$ and $52 \%$, respectively. Patients with primary pulmonary hypertension achieved better long-term survival than patients with Eisenmenger syndrome or pulmonary fibrosis, with a 10 -year survival of $59 \%$ versus $38 \%$ and $24 \%$, respectively (Figure 4). The worst survival was observed in recipients with cystic fibrosis who tested positive for $B$ cepacia before the transplant procedure, with a survival of only $15 \%$ at 10 years (Figure 4). However, the difference in the long-term survival between recipients who tested positive for $B$ cepacia and negative for cystic fibrosis was mainly because of the high rate of septic complications in the $B$ cepacia positive group during the first 6 months after transplantation. Indeed, 15 of 46 recipients died during the first 6 postoperative months in the $B$ cepacia positive group, and all deaths were attributable to $B$ cepacia sepsis. In contrast, only 3 of 78 recipients died of sepsis during the same postoperative period in the $B$ cepacia negative group. After more than 6 months of follow-up, the causes of death

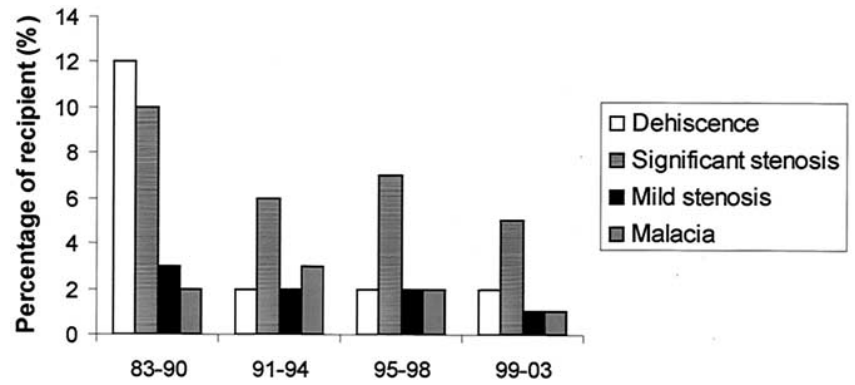

Figure 3. Proportion of patients with airway anastomotic complications decreased over time.

were similar between the $B$ cepacia positive and negative groups, and the survival curves remained parallel.

As a result of the high rate of posttransplant infections in the $B$ cepacia positive group, the immunosuppressive regimen has been reduced and the infection prophylaxis has been enhanced. Since 1995, we have aimed for cyclosporine levels of 250 to $300 \mu \mathrm{g} / \mathrm{mL}$ instead of 250 to $350 \mu \mathrm{g} / \mathrm{mL}$ for the first 6 postoperative months, and azathioprine has been given at a dose of $1 \mathrm{mg} \cdot \mathrm{kg} \cdot \mathrm{d}$ instead of 1.5 to $2.0 \mathrm{mg} \cdot \mathrm{kg}$ . d. Antimicrobial therapy has been extended from 2 to 4 antibiotics including ceftazidime, chloramphenicol, tobramycin, and trimethoprim-sulfamethoxazole. We have also added an aggressive intraoperative irrigation of the airway with a dilute Betadine solution before implantation of the allograft since 1999. We believe that these interventions have improved the outcome in this challenging group of patients and led to a reduction in the number of deaths caused by $B$ cepacia infection to only 2 of 10 patients who underwent transplantation during the past 4 years.

Among patients undergoing bilateral-lung transplantation for emphysema, the long-term survival was significantly worse in those with alpha- 1 antitrypsin deficiency than in those with chronic obstructive pulmonary disease (COPD) without the genetic deficiency (Figure 4). The 

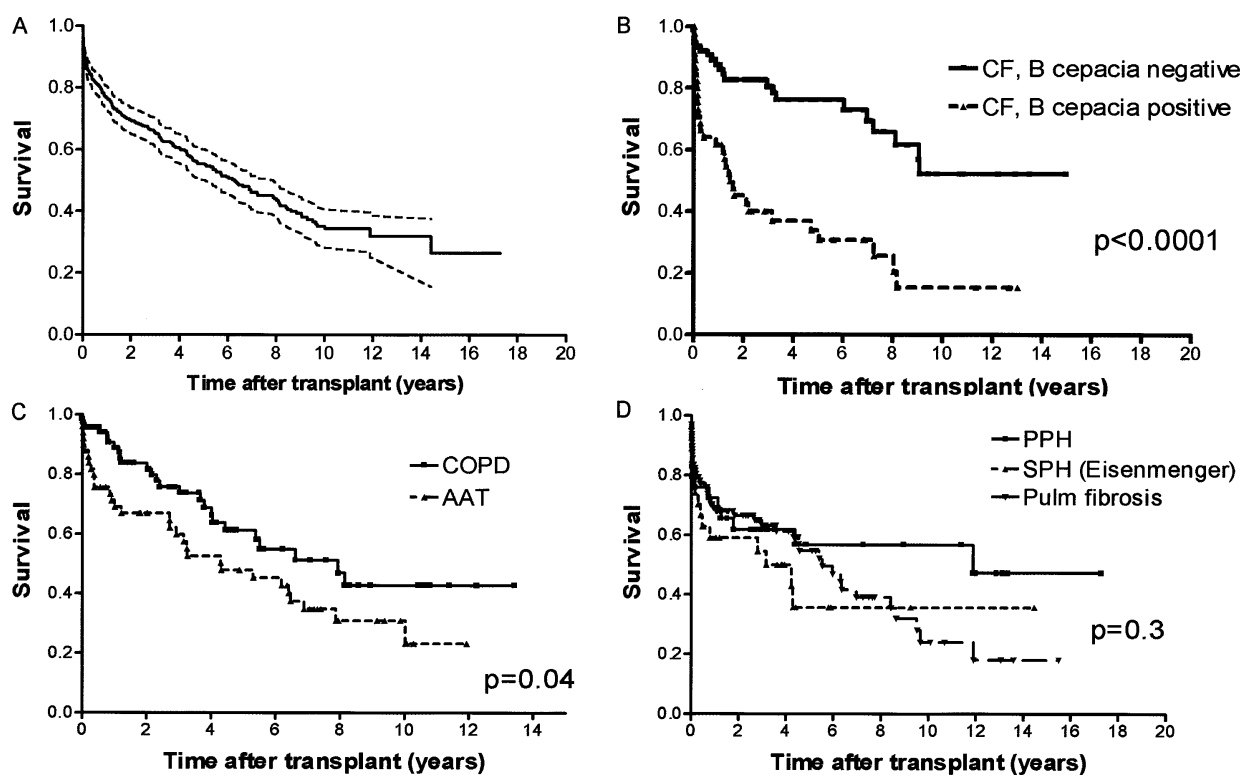

Figure 4. A, Overall survival for 501 recipients (dashed lines: 95\% confidence intervals). B, Survival for recipients with cystic fibrosis was significantly better in those who tested negative for $B$ cepacia when compared with those who tested positive for $\boldsymbol{B}$ cepacia. C, Survival was significantly better in recipients with emphysema undergoing bilateral-lung transplantation for COPD than for recipients with alpha-1 antitrypsin deficiency. D, Survival in recipients undergoing lung transplantation for primary pulmonary hypertension was better than for recipients with Eisenmenger syndrome (severe pulmonary hypertension) or pulmonary fibrosis. CF, Cystic fibrosis; $B$, Burkholderia; $\boldsymbol{P P H}$, primary pulmonary hypertension; $\boldsymbol{S P H}$, severe pulmonary hypertension; $C O P D$, chronic obstructive pulmonary disease; $A A T$, alpha-1 antitrypsin.

TABLE 3. Causes of death in 235 of the 501 recipients who underwent transplantation

\begin{tabular}{lc}
\hline Sepsis & $82(35 \%)$ \\
Chronic graft failure & $68(29 \%)$ \\
Malignancy & $21(9 \%)$ \\
Cardiac event & $15(6 \%)$ \\
Primary graft failure & $12(4 \%)$ \\
Pulmonary emboli & $5(2 \%)$ \\
Other & $32(15 \%)$ \\
\hline
\end{tabular}

10 -year survival was $23 \%$ in recipients with alpha- 1 antitrypsin deficiency, whereas it was $43 \%$ in the remaining recipients with emphysema $(P=.04)$.

Recipient age and use of CPB had no significant impact on the long-term outcome of patients after lung transplantation. The effect of time of transplant also had no significant impact on long-term outcome. The long-term survival tended to be better in patients undergoing bilateral-lung transplants than in patients undergoing single-lung transplants $(P=.07)$. However, this effect disappeared when the type of transplant was analyzed separately for emphysema, pulmonary fibrosis, pulmonary hypertension, and other types of parenchymal diseases.

The causes of death are summarized in Table 3. Malig- nancy included mainly lymphoma $(\mathrm{n}=10)$ and less frequently solid-organ tumors such as lung carcinoma $(\mathrm{n}=3)$, liver carcinoma $(n=2)$, and colon, breast, esophagus, and renal carcinomas $(\mathrm{n}=1 \mathrm{each})$. Skin tumors were the cause of death in 2 recipients.

Sepsis was the main cause of death during the first 6 months after transplantation; chronic graft failure and bronchiolitis obliterans syndrome (BOS) became the predominant causes of death thereafter (Table 4). Despite the risk of sepsis in the group with $B$ cepacia positive cystic fibrosis during the first 6 months after transplant, the rate of death from BOS is high in those surviving more than 6 months $(56 \%)$ and not significantly different from recipients with $B$ cepacia negative cystic fibrosis $(60 \%)$. The susceptibility to septic complications seems to differ in recipients with emphysema according to whether they are alpha- 1 antitrypsin deficient or not. Indeed, 4 of 88 patients who underwent transplantation for COPD died during the first 6 months after transplantation, and only 1 of them died of sepsis. In contrast, 16 of 63 patients with alpha-1 antitrypsin deficiency died within 6 months of surgery, and 8 of them died of sepsis. It is interesting that beyond 6 months, patients with alpha-1 antitrypsin deficiency still exhibited a high susceptibility to septic complications (45\% of the deaths were attributable to sepsis), whereas the death rate from 
sepsis was $25 \%$ or less for other pulmonary diseases (Table 4).

The rate of septic complications in the alpha- 1 antitrypsin group was not related to the occurrence of BOS. Indeed, the incidence of BOS in patients surviving more than 6 months was not significantly different between those who tested negative (37\%) or positive (56\%) for B cepacia cystic fibrosis, or among those who had primary pulmonary hypertension (44\%), COPD (39\%), alpha-1 antitrypsin deficiency $(46 \%)$, or pulmonary fibrosis $(25 \%)$. Thus, the incidence of BOS in recipients deficient in alpha- 1 antitrypsin was similar to other groups, but the proportion of deaths from BOS was smaller because there were more septic deaths (Table 4). Among all patients surviving more than 6 months, $11 \%$ (5/47) died of BOS in the alpha- 1 antitrypsin deficient group versus $18 \%(65 / 365)$ in the remaining recipient groups $(P=.2)$. However, $21 \%(10 / 47)$ of recipients with alpha- 1 antitrypsin deficiency died of late sepsis versus only $6 \%(21 / 365)$ in the remaining recipients $(P=.0001)$.

\section{Discussion}

The Toronto Lung Transplant Program was started in the 1970s. After several years of research, the first successful single-lung transplant was performed in 1983 in a patient with pulmonary fibrosis. That patient eventually died 7 years later of BOS and renal failure. In 1986, the first double-lung transplant was performed in a patient with emphysema secondary to alpha-1 antitrypsin deficiency. The patient remained well and active for 15 years after the transplant before dying suddenly of a subarachnoid hemorrhage.

After this initial development, lung transplantation has had increasing success and become the mainstay of therapy for many end-stage pulmonary diseases worldwide. The indications have been extended from patients with pulmonary fibrosis and obstructive lung disease to patients with infectious processes and pulmonary hypertension. Recipient selection has also been extended to selected higher risk recipients, such as patients who are ventilator-dependent or who have end-stage lung disease associated with other chronic diseases (including coronary artery disease and liver dysfunction). The age limit has also progressively been extended to patients 65 years of age and older.

Our program favors the use of bilateral-lung transplantation. The international experience and specific reports from large centers have suggested that long-term survival is significantly better in emphysematous recipients undergoing bilateral-lung transplant rather than single-lung transplant. ${ }^{9,10}$ In addition, we find recipients of bilateral-lung transplants to be easier to manage during the postoperative period if prolonged mechanical ventilation is required. Single-lung transplantation is generally performed when only 1 lung is available because the other lung is either used by
TABLE 4. Causes of death according to recipient lung disease and time after transplant

\begin{tabular}{|c|c|c|}
\hline & Time aft & nsplant \\
\hline & $<6$ mo & $>6 \mathrm{mo}$ \\
\hline Pulmonary fibrosis $(\mathrm{n}=97$ ) & & \\
\hline Total number of deaths & 22 & 29 \\
\hline Death from BOS & $1(5 \%)$ & $13(45 \%)$ \\
\hline Death from sepsis & $11(50 \%)$ & $4(14 \%)$ \\
\hline Death from other causes & $10(45 \%)$ & $12(41 \%)$ \\
\hline $\operatorname{COPD}(\mathrm{n}=88)$ & & \\
\hline Total number of deaths & 4 & 29 \\
\hline Death from BOS & $0(0 \%)$ & $16(55 \%)$ \\
\hline Death from sepsis & $1(25 \%)$ & $4(14 \%)$ \\
\hline Death from other causes & $3(75 \%)$ & $9(31 \%)$ \\
\hline $\begin{array}{l}\text { Alpha-1 antitrypsin deficiency ( } \\
\quad=63)\end{array}$ & & \\
\hline Total number of deaths & 16 & 22 \\
\hline Death from BOS & $0(0 \%)$ & $5(23 \%)$ \\
\hline Death from sepsis & $8(50 \%)$ & $10(45 \%)$ \\
\hline Death from other causes & $8(50 \%)$ & $7(32 \%)$ \\
\hline Pulmonary hypertension $(\mathrm{n}=$ & & \\
\hline Total number of deaths & 17 & 11 \\
\hline Death from BOS & $1(6 \%)$ & $7(64 \%)$ \\
\hline Death from sepsis & $8(47 \%)$ & $0(0 \%)$ \\
\hline Death from other causes & $8(47 \%)$ & $4(36 \%)$ \\
\hline $\begin{array}{l}\text { Cystic fibrosis, B cepacia } \\
\text { negative }(\mathrm{n}=78)\end{array}$ & & \\
\hline Total number of deaths & 6 & 15 \\
\hline Death from BOS. & $0(0 \%)$ & $9(60 \%)$ \\
\hline Death from sepsis & $3(50 \%)$ & $3(20 \%)$ \\
\hline Death from other causes & $3(50 \%)$ & $3(20 \%)$ \\
\hline $\begin{array}{l}\text { Cystic fibrosis, B cepacia } \\
\text { positive }(n=46)\end{array}$ & & \\
\hline Total number of deaths & 15 & 16 \\
\hline Death from BOS & $0(0 \%)$ & $9(56 \%)$ \\
\hline Death from sepsis & $15(100 \%)$ & $4(25 \%)$ \\
\hline Death from other causes & $0(0 \%)$ & $3(19 \%)$ \\
\hline $\begin{array}{l}\text { Other causes of end-stage lun } \\
\text { disease }(n=77)\end{array}$ & & \\
\hline Total number of deaths & 9 & 24 \\
\hline Death from BOS & $0(0 \%)$ & $11(46 \%)$ \\
\hline Death from sepsis & $5(55 \%)$ & $6(25 \%)$ \\
\hline Death from other causes & $4(45 \%)$ & $7(29 \%)$ \\
\hline
\end{tabular}

$B O S$, Bronchiolitis obliterans syndrome; COPD, chronic obstructive pulmonary disease.

another program or is compromised because of localized trauma or infection. Our preference for bilateral-lung transplantation, even for patients with COPD, may explain the good long-term outcome observed for these patients in our series (10-year survival of 43\%).

The overall 10 -year survival is $35 \%$ in our series. However, some groups of recipients achieved better long-term survival. For instance, the 10-year survival was more than $50 \%$ for recipients with primary pulmonary hypertension and $B$ cepacia negative cystic fibrosis, whereas it was only $24 \%$ for recipients with pulmonary fibrosis. Although several groups have reported excellent results in recipients with 
cystic fibrosis when compared with other lung transplant recipients, very few have reported a long-term survival more than $50 \%$ for recipients with primary pulmonary hypertension. ${ }^{11-13}$ The reason for this good long-term outcome remains speculative. One reason may relate to the quality of the lung donor used. Indeed, recipients with primary pulmonary hypertension have an increased risk of postoperative complications, and extended or marginal donors are less frequently used for this group of recipients. We and others have also observed that the pathologic lesions leading to primary pulmonary hypertension can regress after singlelung transplantation because of preferential blood flow to the transplanted lung and hemodynamic unloading of the native lung. ${ }^{14,15}$ Thus, when the transplanted lung progressively fails, the native lung may be able to resume some lung function and keep the recipient alive.

In our experience with 501 patients, the majority died of sepsis (35\%) and chronic graft failure (29\%). However, the cause of death varied with time after transplant. Overall, sepsis was the predominant cause of death in the first 6 months after the transplant procedure, whereas chronic graft failure was the main cause of death after 6 months. The only exception was patients with a preoperative diagnosis of alpha-1 antitrypsin deficiency who continued to be predisposed to sepsis-related mortality, even beyond 6 months after transplant. Sepsis was predominantly caused by bacterial infection both from pulmonary and extra pulmonary origin. Of note, in the alpha- 1 antitrypsin group, $25 \%$ of patients had an episode of sepsis of abdominal origin while followed by the lung transplant team, and in $17 \%$ of them it contributed to their deaths. In contrast, in the remaining patients with emphysema, who were not alpha- 1 antitrypsin deficient, abdominal sepsis occurred in only $8 \%$ of patients and contributed to only $1 \%$ of deaths.

Patients with alpha-1 antitrypsin deficiency also had a higher early postoperative mortality rate and a shorter longterm survival than recipients with emphysema without alpha-1 antitrypsin deficiency. This finding has not been reported and may be the result of an increased level of interleukin- 8 and increased chemotactic activity of neutrophils in the lung tissue of patients with alpha- 1 antitrypsin deficiency. ${ }^{16}$ Both interleukin-8 and neutrophil activation have been shown to be associated with an increased risk of acute graft dysfunction after lung transplantation. ${ }^{17,18}$ In addition, lung transplant recipients deficient in alpha- 1 antitrypsin have been shown to have lower levels of antiprotease defenses during reperfusion injury, acute rejection, and infection, and the difficulty in clearing up neutrophils from the lung parenchyma may eventually be associated with increased mortality in the long term. ${ }^{19}$

\section{Conclusion}

Lung transplantation offers long-term survival in many patients with end-stage lung disease. The long-term survival was best in patients who underwent transplantation for primary pulmonary hypertension and cystic fibrosis $(\mathrm{B} \mathrm{Ce}$ pacia negative), with a 10 -year survival greater than $50 \%$. The lowest survivals were observed in recipients with pulmonary fibrosis, alpha-1 antitrypsin deficiency, and B cepacia positive cystic fibrosis, with 10 -year survivals of $24 \%$, $23 \%$, and $15 \%$, respectively, after the transplant procedure. The primary cause of death was sepsis during the initial 6 months after surgery and chronic graft dysfunction later on. Sepsis, however, remained an important cause of death in patients with alpha-1 antitrypsin deficiency even beyond 6 months after transplantation. Challenges for the future will be to reduce the incidence of infection and to prevent the development of chronic graft dysfunction or obliterative bronchiolitis.

\section{References}

1. Hardy JD. The first lung transplant in man (1963) and the first heart transplant in man (1964). Transplant Proc. 1999;31:25-9.

2. Veith FJ, Kamholz SL, Mollenkopf FP, Montefusco CM. Lung transplantation 1983. Transplantation. 1983;35:271-8.

3. Unilateral lung transplantation for pulmonary fibrosis. Toronto Lung Transplant Group. N Engl J Med. 1986;314:1140-5.

4. Patterson GA, Cooper JD, Dark JH, Jones MT. Experimental and clinical double lung transplantation. J Thorac Cardiovasc Surg. 1988; 95:70-4.

5. Pasque MK, Cooper JD, Kaiser LR, Haydock DA, Triantafillou A, Trulock EP. Improved technique for bilateral lung transplantation: rationale and initial clinical experience. Ann Thorac Surg. 1990;49: 785-91.

6. Maurer JR, Frost AE, Estenne M, Higenbottam T, Glanville AR. International guidelines for the selection of lung transplant candidates. The International Society for Heart and Lung Transplantation, the American Thoracic Society, the American Society of Transplant Physicians, the European Respiratory Society. J Heart Lung Transplant. 1998;17:703-9.

7. De Perrot M, Liu M, Waddell TK, Keshavjee S. Ischemia-reperfusioninduced lung injury. Am J Respir Crit Care Med. 2003;167:490-511.

8. De Perrot M, Keshavjee S. Everting mattress running suture: an improved technique of atrial anastomosis in human lung transplantation. Ann Thorac Surg. 2002;73:1663-4.

9. Hertz M, Taylor D, Trulock E, Boucek M, Mohacsi P, Edwards L, et al. The registry of the international society for heart and lung transplantation: nineteenth official report-2002. J Heart Lung Transplant. 2002;21:950.

10. Cassivi SD, Meyers BF, Battafarano RJ, Guthrie TJ, Trulock EP, Lynch JP, et al. Thirteen-year experience in lung transplantation for emphysema. Ann Thorac Surg. 2002;74:1663-9.

11. Egan TM, Detterbeck FC, Mill MR, Bleiweis MS, Aris R, Paradowski $\mathrm{L}$, et al. Long term results of lung transplantation for cystic fibrosis. Eur J Cardiothorac Surg. 2002;22:602-9.

12. Harringer W, Wiebe K, Struber M, Franke U, Niedermeyer J, Fabel H, et al. Lung transplantation-10-year experience. Eur J Cardiothorac Surg. 1999;16:546-54.

13. Macchiarini P, Ladurie FL, Cerrina J, Fadel E, Chapelier A, Dartevelle P. Clamshell or sternotomy for double lung or heart-lung transplantation? Eur J Cardiothorac Surg. 1999;15:333-9.

14. Levy NT, Liapis H, Eisenberg PR, Botney MD, Trulock EP. Pathologic regression of primary pulmonary hypertension in left native lung following right single-lung transplantation. J Heart Lung Transplant. 2001;20:381-4.

15. O'Blenes SB, Fischer S, McIntyre B, Keshavjee S, Rabinovitch M. Hemodynamic unloading leads to regression of pulmonary vascular disease in rats. J Thorac Cardiovasc Surg. 2001;121:279-89.

16. Woolhouse IS, Bayley DL, Stockley RA. Sputum chemotactic activity 
in chronic obstructive pulmonary disease: effect of alpha(1)-antitrypsin deficiency and the role of leukotriene $\mathrm{B}(4)$ and interleukin 8 . Thorax. 2002;57:709-14.

17. De Perrot M, Sekine Y, Fischer S, Waddell TK, McRae K, Liu M, et al. Interleukin-8 release during early reperfusion predicts graft function in human lung transplantation. Am J Respir Crit Care Med. 2002;165:211-5.

18. Fisher AJ, Donnelly SC, Hirani N, Haslett C, Strieter RM, Dark JH, et al. Elevated levels of interleukin- 8 in donor lungs is associated with early graft failure after lung transplantation. Am J Respir Crit Care Med. 2001;163:259-65.

19. Meyer KC, Nunley DR, Dauber JH, Iacono AT, Keenan RJ, Cornwell $\mathrm{RD}$, et al. Neutrophils, unopposed neutrophil elastase, and alpha1antiprotease defenses following human lung transplantation. Am J Respir Crit Care Med. 2001;164:97-102.

\section{Discussion}

Dr Thomas M. Egan (Chapel Hill, NC). In many respects your results agree with those of the larger International Society for Heart and Lung Transplantation (ISHLT) data set. It is discouraging that a 70 year old with stage I lung cancer has a better prognosis today than a 25 year old undergoing a lung transplant despite 20 years of experience. It is also discouraging that there are not nearly enough lungs to go around to treat everyone with end-stage lung disease.

In your series, the 2 main causes of death were sepsis related to broad, nonspecific immunosuppression and BOS, an inevitable process that we do not seem to understand. Your observation that patients with pulmonary hypertension have a poor earlier prognosis is corroborated by the ISHLT registry. This registry also confirms your observation that patients with alpha-1 antitrypsin deficiency do not do as well early, but in the ISHLT registry they have a better long-term survival than older patients with COPD, unlike in your series.

I have a few questions. With the 521 transplants that you have performed, have you had the opportunity to perform any univariate or multivariate analyses to identify risk factors for death? Can you explain why your patients with COPD had a 5-year survival that was not so good, almost $20 \%$ better than reported in the ISHLT registry or south of the border? Could it be the colder Canadian air? How many patients died while on your waiting list during this interval? And can you clarify for us the criteria for distribution of lungs in Canada? The United Network for Organ Sharing is currently struggling to devise an organ distribution algorithm in the United States that places less emphasis on time waiting and more emphasis on severity of illness. How do you determine who is offered a lung when lungs become available?

Finally, I applaud your group's long tradition of taking clinical problems to the laboratory to come up with solutions. I would submit that there are 3 challenges for future lung transplant surgeons: the shortage of suitable lungs; potent, nonspecific immunosuppression that causes infection; and the scourge of BOS. So my last question is, from your perspective of the last 20 years, how do you see us finding solutions to these issues in the next 20 years?
Dr de Perrot. We have not yet performed multivariate analyses, but we are still retrieving some of the data for other variables, such as CMV and donor factors for each of the recipients.

The survival in the COPD group is surprisingly good. However, the reason is not very clear. One of them could be the long tradition of transplantation in Toronto, which may explain some of those results.

An unexpected finding comes from the differences between the groups with COPD and alpha-1 antitrypsin deficiency. The group with alpha-1 antitrypsin deficiency did worse during the earlier postoperative period and later on as well. Despite the fact that the operation is relatively similar, there may be some reasons associated with the alpha- 1 antitrypsin deficiency that may explain these findings.

The number of patients who die while on the waiting list is low. Our current waiting time is approximately 3 to 4 months. During the last few years, we have expanded the number of donors used. Earlier on, 10 years ago, approximately $15 \%$ to $20 \%$ of the lung donors were used, and this has increased to $40 \%$ last year. So the fact that we have been more aggressive with these donors has decreased the waiting time, and it has certainly helped to decrease the number of deaths on the waiting list.

The criteria for donor lung distribution in Canada is, as you know, different from the United States. The donors are attributed alternatively to each center, and the center determines who should receive the organ. Certainly the sickness of each patient is taken into consideration to determine which recipient should get the lungs. This may also affect the number of deaths on the waiting list.

The focus for the next decade or 2 certainly will be on the relatively poor long-term survival. The first 10 years of successful lung transplantation focused on technical issues, and the last 10 years were associated with improvement in the methods of lung preservation. So possibly in the next decade we will see improvement in the prevention and treatment of BOS.

Dr Si M. Pham (Miami, Fla). During the last 20 years, there have been quite a few changes in the use of immunosuppressive agents. The 2 major complications you report, infection and chronic rejection, can be partially attributed to the immunosuppression protocol used. Has the immunosuppression protocol at your center changed during the last 20 years?

Dr de Perrot. It certainly has. Whereas initially most of these patients received induction therapy, no systematic induction therapy has been used in the past 6 to 8 years in Toronto. I think that the most significant change has occurred in the patients who test positive for $B$ cepacia cystic fibrosis. These patients have had lower immunosuppression for the last several years and received broad-spectrum antibiotics. This was associated with tracheal washing during the surgery. These changes were associated with an improvement in the number of deaths resulting from sepsis, which decreased from approximately $40 \%$ in the early period to approximately $20 \%$ in the past 4 years or so for the B cepacia group. 